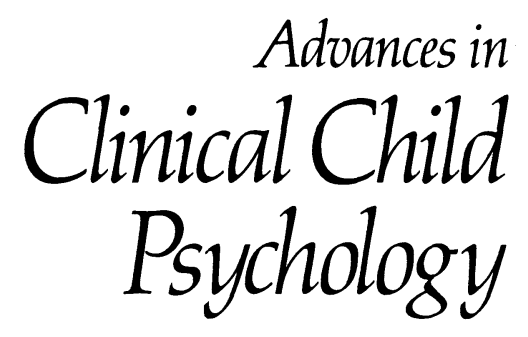

Volume 4 


\section{ADVANCES IN CLINICAL CHILD PSYCHOLOGY}

Advisory Editors:

DONNA M. GELFAND, University of Utah

JOHN C. MASTERS, University of Minnesota

TODD R. RISLEY, University of Kansas

DAVID L. ROSENHAN, Stanford University

ALAN O. ROSS, State University of New York at Stony Brook

A Continuation Order Plan is available for this series. A continuation order will bring delivery of each new volume immediately upon publication. Volumes are billed only upon actual shipment. For further information please contact the publisher. 


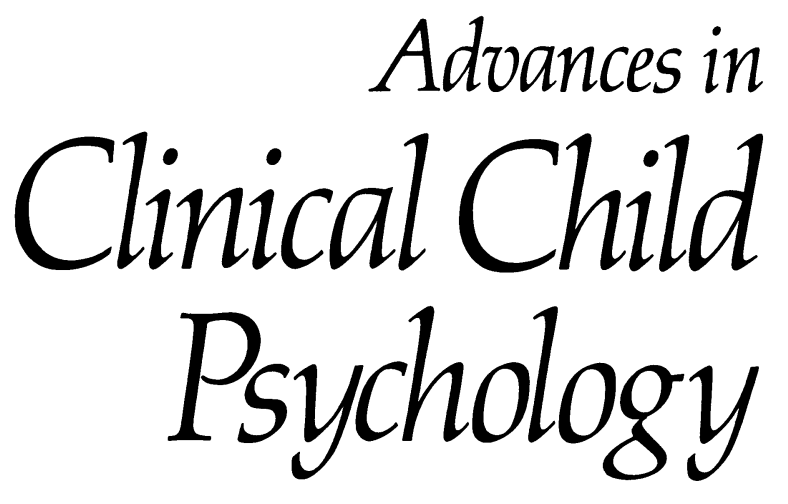

\title{
Volume 4
}

\author{
Edited by \\ Benjamin B. Lahey \\ University of Georgia \\ Athens, Georgia \\ and \\ Alan E. Kazdin
}

Western Psychiatric Institute and Clinic University of Pittsburgh School of Medicine Pittsburgh, Pennsylvania 
The Library of Congress cataloged the first volume of this title as follows:

Advances in clinical child psychology. v. 1-

New York, Plenum Press, c1977-

v. ill. $24 \mathrm{~cm}$.

Key title: Advances in clinical child psychology. ISSN 0149-4732

1. Clinical psychology-Collected works. 2. Child psychology-Collected works. 3. Child psychotherapy-Collected works.

()1981 Plenum Press, New York Softcover reprint of the hardcover 1st edition 1981

A Division of Plenum Publishing Corporation 233 Spring Street, New York, N.Y. 10013

All rights reserved

No part of this book may be reproduced, stored in a retrieval system, or transmitted, in any form or by any means, electronic, mechanical, photocopying, microfilming, recording, or otherwise, without written permission from the Publisher 
This series is dedicated to the children of the world, especially MEGAN, EDWARD, ERIN, NICOLE, and MICHELLE 


\section{Contributors}

Jon S. Bailey

R. Christopher Barden

Rand D. Conger

Elyse Schwartz Felleman

Sharon L. Foster

James J. Fox

Brandon F. Greene

Raymond P. Harrison

John J. Horan

Philip C. Kendall

Kenneth K. Kidd
Department of Psychology, Florida State University, Tallahassee, Florida

Department of Psychology, Southern Methodist University, Dallas, Texas

Department of Sociology, University of Georgia, Athens, Georgia

Department of Psychology, Vanderbilt University, Nashville, Tennessee

Department of Psychology, West Virginia University, Morgantown, West Virginia

Department of Special Education, Vanderbilt University, Nashville, Tennessee

Department of Psychology, Southern Illinois University, Carbondale, Illinois

Department of Psychiatry, Center for Cognitive Therapy, University of Pennsylvania, Philadelphia, Pennsylvania

Division of Counseling and Educational Psychology, Pennsylvania State University, University Park, Pennsylvania

Department of Psychology, University of Minnesota, Minneapolis, Minnesota

Department of Human Genetics, Yale University School of Medicine, New Haven, Connecticut 
John C. Masters

Larry Michelson

David L. Pauls

Wendy L. Ritchey

Phillip S. Strain

William H. Yeaton

William Yule
Department of Psychology, Vanderbilt University, Nashville, Tennessee

Department of Psychiatry, Western Psychiatric Institute and Clinic, University of Pittsburgh, School of Medicine, Pittsburgh, Pennsylvania

Department of Human Genetics, Yale University School of Medicine, New Haven, Connecticut

Department of Psychology, West Virginia University, Morgantown, West Virginia

Department of Psychiatry, University of Pittsburgh, School of Medicine, Western Psychiatric Institute and Clinic, Pittsburgh, Pennsylvania

Institute for Social Research/CRUSK, University of Michigan, Ann Arbor, Michigan

Department of Psychology, University of London, Institute of Psychiatry, De Crespigny Park, London, England 


\section{Preface}

Advances in Clinical Child Psychology is a serial publication designed to provide researchers and clinicians with a medium for discussing new and innovative approaches to the problems of children. In this fourth volume, a group of highly distinguished authors have described advancing knowledge in a number of critical areas of applied child psychology. These include childhood depression, drug abuse, social skills deficits, community-living skills, the genetics of childhood behavior disorders, and affective states in children. In addition, major statements on new approaches to the assessment of dysfunctional family systems and the social skills of children, as well as the increasingly important methodology of epidemiology, are included in this volume. These chapters provide a synopsis of many of the most important advances in the field of clinical child psychology.

The quality of a series of this sort is, of course, due to the quality of the contributing authors. We feel very fortunate indeed, therefore, to have been able to entice such a distinguished group of authors to contribute to this volume. We are also most appreciative of the guidance and assistance of the consulting editors who provided us with ideas for chapter topics and authors and who carefully reviewed and edited each chapter. We also express our hearty thanks to Leonard R. Pace of Plenum whose expertise and support has always been generously given.

BENJAMin B. LAHEY

Alan E. Kazdin 


\section{Contents}

The Epidemiology of Child Psychopathology

1

William Yule

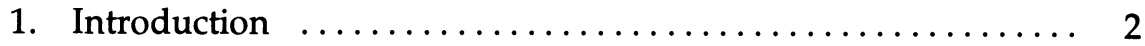

1.1. Definition of Epidemiology $\ldots \ldots \ldots \ldots \ldots \ldots \ldots \ldots, 2$

1.2. Incidence and Prevalence $\ldots \ldots \ldots \ldots \ldots \ldots \ldots \ldots, 3$

2. What Is a "Case?" .......................... 5

2.1. A Developmental Perspective ................ 5

2.2. Situation Specificity $\ldots \ldots \ldots \ldots \ldots \ldots \ldots \ldots \ldots \ldots \ldots$

2.3. Defect, Disability, and Handicap .............. 7

2.4. The Perspective of Significant Others $\ldots \ldots \ldots \ldots \ldots, 8$

2.5. One Working Solution $\ldots \ldots \ldots \ldots \ldots \ldots \ldots \ldots, 9$

3. Classification and Categorization $\ldots \ldots \ldots \ldots \ldots \ldots \ldots \ldots \ldots$

3.1. Characteristics of a Good System of Classification ..... 12

3.2. Development of Systems of Classification ........... 14

3.3. The World Health Organization's International Classification of Diseases-9 .................. 17

3.4. Diagnostic and Statistical Manual, Third Edition (DSM-III) ............................. 19

3.5. Critique of ICD-9 and DSM-III $\ldots \ldots \ldots \ldots \ldots \ldots \ldots 20$

3.6. Multivariate Approaches $\ldots \ldots \ldots \ldots \ldots \ldots \ldots \ldots, 25$

4. Results of Epidemiological Studies $\ldots \ldots \ldots \ldots \ldots \ldots \ldots \ldots$

4.1. The Isle of Wight Studies $\ldots \ldots \ldots \ldots \ldots \ldots \ldots \ldots, 27$

4.2. Studies of Adolescents ...................... 34

4.3. The Inner-London Comparative Study $\ldots \ldots \ldots \ldots \ldots 36$

4.4. Preschool Behavior Problems ................. 36

5. Prevalence Rates of Clinical Psychiatric Syndromes ........ 37

5.1. Attention Deficit Disorder with Hyperactivity or

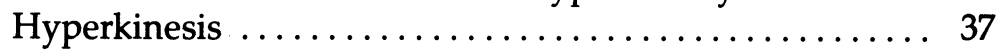

5.2. Conduct Disorders $\ldots \ldots \ldots \ldots \ldots \ldots \ldots \ldots, \ldots$

5.3. Emotional Disorders $\ldots \ldots \ldots \ldots \ldots \ldots \ldots \ldots \ldots \ldots$

5.4. Other Disorders $\ldots \ldots \ldots \ldots \ldots \ldots \ldots \ldots \ldots \ldots \ldots \ldots$

5.5. Eating Disorders .............................. 39

5.6. Stereotyped Movement Disorders ............. 40

5.7. Other Disorders with Physical Manifestations $\ldots \ldots \ldots .40$ 
5.8. Pervasive Development Disorders $\ldots \ldots \ldots \ldots \ldots \ldots \ldots 41$

5.9. Specific Development Disorders $\ldots \ldots \ldots \ldots \ldots \ldots \ldots, 42$

5.10. Comment ............................. 42

6. Methodological Problems $\ldots \ldots \ldots \ldots \ldots \ldots \ldots \ldots \ldots, 43$

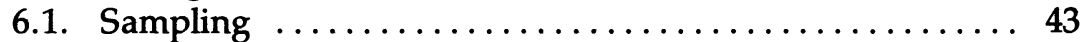

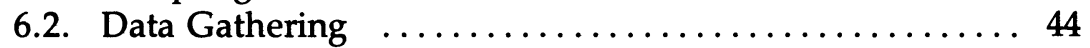

6.3. Correlation and Causality .................. 45

7. Toward a Psychological Approach to Childhood

Psychopathology ............................ 45

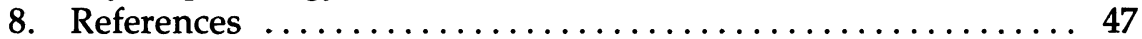

Cognitive-Behavioral Interventions with

Children

Philip C. Kendall

1. Relation to Adult Cognitive-Behavioral Therapy $\ldots \ldots \ldots \ldots . \ldots 3$

2. A Definition? ........................... 55

3. Cognitive-Behavioral Strategies for Teaching Self-Control ... 57

3.1. Training Tasks $\ldots \ldots \ldots \ldots \ldots \ldots \ldots \ldots \ldots \ldots \ldots \ldots \ldots \ldots$

3.2. Verbal Self-Instructions $\ldots \ldots \ldots \ldots \ldots \ldots \ldots \ldots \ldots, 58$

3.3. Modeling ............................ 61

3.4. Contingencies: Response-Cost, Self-Reward, and

Earning Rewards ....................... 61

3.5. Role-Playing and Self-Evaluation $\ldots \ldots \ldots \ldots \ldots \ldots, 63$

3.6. Research Evaluations ..................... 65

4. Social Perspective-Taking (Role-Taking) Training $\ldots \ldots \ldots \ldots 75$

4.1. Research Outcomes ...................... 77

5. Interpersonal Problem-Solving Training $\ldots \ldots \ldots \ldots \ldots \ldots . \ldots 9$

5.1. Research Outcomes .................... 80

6. Consistencies across Cognitive-Behavioral Interventions with

Children ................................. 82

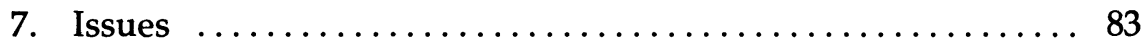

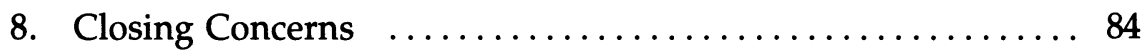

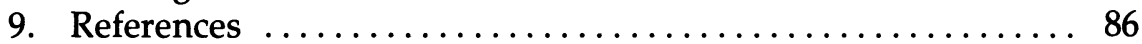

Experimental Studies of Affective States in Children

John C. Masters, Elyse Schwartz Felleman, and $R$. Christopher Barden

1. Introduction $\ldots \ldots \ldots \ldots \ldots \ldots \ldots \ldots \ldots \ldots \ldots \ldots \ldots \ldots \ldots$

2. Theories of Emotion $\ldots \ldots \ldots \ldots \ldots \ldots \ldots \ldots \ldots \ldots \ldots \ldots \ldots$ 
3. Procedures for the Experimental Induction of Emotional Cognitive States in Children and Adults .............. 92

4. Validation of Experimental Affect Induction Procedures ...... 95

5. Experiential Determinants of Affective States $\ldots \ldots \ldots \ldots .98$

5.1. Success/Failure ........................ 99

5.2. Experiences of Nurturance $\ldots \ldots \ldots \ldots \ldots \ldots \ldots \ldots \ldots \ldots$

5.3. Reward/Reinforcement and Punishment .......... 100

5.4. Vicarious Experience: The Observation of Emotion in Others .............................. 102

5.5. Summary: Understanding Affect-Inducing Experiences .............................. 102

6. Behavioral and Cognitive Consequences of Affective States .. 103

6.1. Generosity .............................. 103

6.2. Self-Gratification .......................... 105

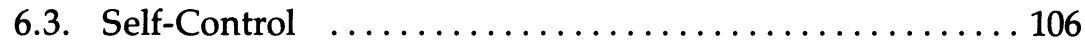

6.4. Aggression ........................... 107

6.5. Spontaneous Coping Responses $\ldots \ldots \ldots \ldots \ldots \ldots \ldots 107$

6.6. Cognitive Processing $\ldots \ldots \ldots \ldots \ldots \ldots \ldots \ldots \ldots \ldots \ldots$

7. Factors Mediating the Relation between Affect and Behavior ............................... 110

8. Experimental Studies of Self-Implemented and Externally-Implemented Cognitive and Behavioral Procedures to Alter Affective States ......................... 112

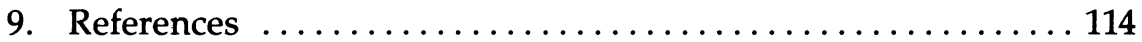

Social-Skills Assessment of Children Larry Michelson, Sharon L. Foster, and Wendy L. Ritchey

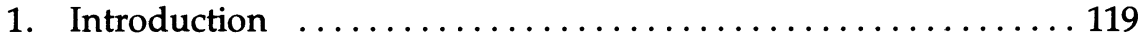

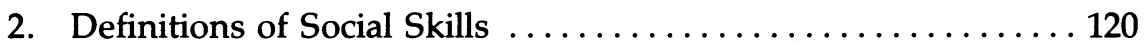

3. Dimensions of Analysis ........................ 122

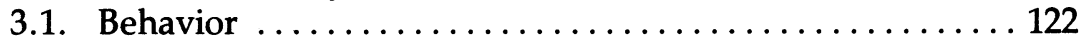

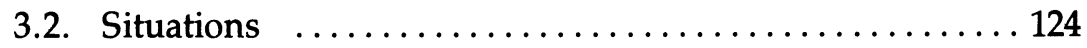

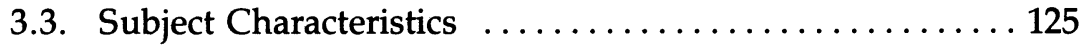

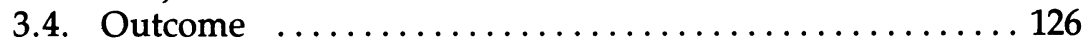

4. Methods of Assessment ....................... 131

4.1. Role-Play Assessment $\ldots \ldots \ldots \ldots \ldots \ldots \ldots \ldots \ldots \ldots \ldots$

4.2. Ratings by Significant Others $\ldots \ldots \ldots \ldots \ldots \ldots \ldots \ldots \ldots$

4.3. Self-Report Measures . .................... 147

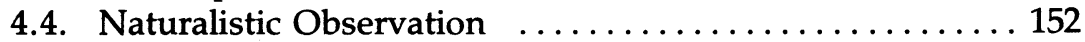

5. Conclusions and Future Directions $\ldots \ldots \ldots \ldots \ldots \ldots \ldots \ldots$

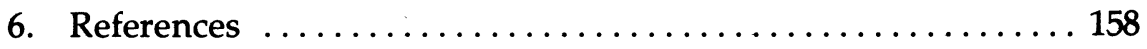


Peers as Behavior Change Agents for Withdrawn Classmates

Phillip S. Strain and James J. Fox

\section{5}

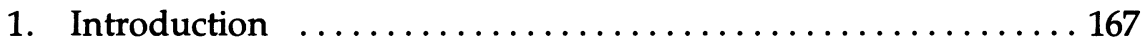

2. Behavioral Treatment of Preschool

Social Withdrawal .............................. 169

3. Peer-Mediated Intervention Strategies $\ldots \ldots \ldots \ldots \ldots \ldots \ldots 172$

3.1. Prompting and Reinforcement ............... 172

3.2. Peer Social Initiations $\ldots \ldots \ldots \ldots \ldots \ldots \ldots \ldots \ldots \ldots \ldots$

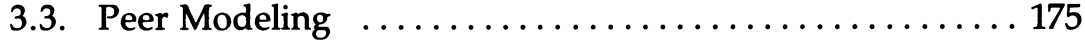

3.4. Incidental Peer Influence $\ldots \ldots \ldots \ldots \ldots \ldots \ldots \ldots \ldots \ldots \ldots$

3.5. Conclusions ............................. 180

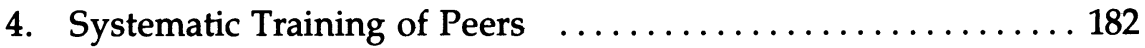

5. Assessment and Treatment Evaluation $\ldots \ldots \ldots \ldots \ldots \ldots \ldots 185$

6. Generalization of Treatment Effects ................ 189

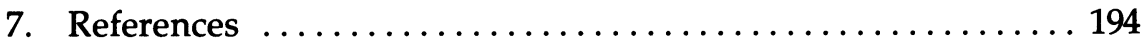

The Assessment of Dysfunctional Family Systems

Rand D. Conger

1. Overview ......................................... 199

1.1. Behavioral Assessment ...................... 200

1.2. Systems Analysis . ....................... 201

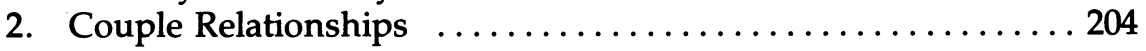

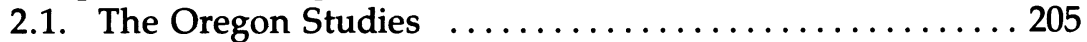

2.2. The Illinois and Indiana Studies $\ldots \ldots \ldots \ldots \ldots \ldots . \ldots 211$

2.3. Discussion and Conclusions $\ldots \ldots \ldots \ldots \ldots \ldots \ldots . . \ldots 216$

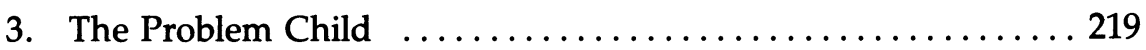

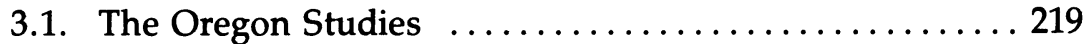

3.2. The Georgia Studies . .................... 223

3.3. The Tennessee Studies ..................... 226

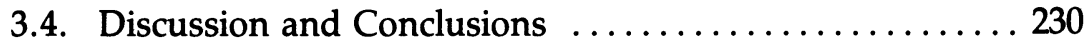

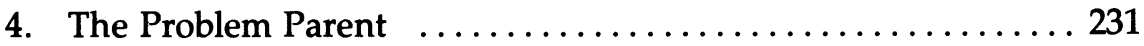

4.1. The Oregon Studies $\ldots \ldots \ldots \ldots \ldots \ldots \ldots \ldots \ldots \ldots . \ldots \ldots \ldots$

4.2. The Pennsylvania Studies .................. 234

4.3. Discussion and Conclusions $\ldots \ldots \ldots \ldots \ldots \ldots \ldots \ldots 237$

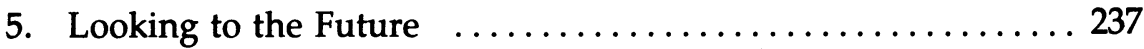

6. References ............................... 239 
Behavioral Community Psychology: Strategies and Tactics for Teaching Community Skills to Children and Adolescents William H. Yeaton, Brandon F. Greene, and Jon S. Bailey

1. Representative Contemporary Research $\ldots \ldots \ldots \ldots \ldots . \ldots 244$

1.1. Nutrition ............................. 244

1.2. Medical Care ............................. 247

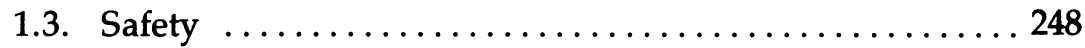

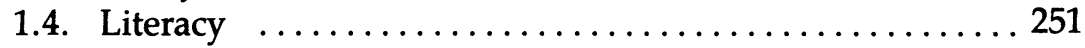

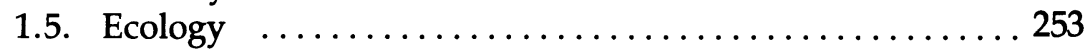

1.6. Work Skills ............................. 255

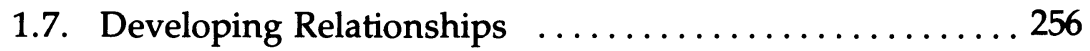

1.8. Leisure and Recreation ...................... 258

1.9. Consumerism ........................... 259

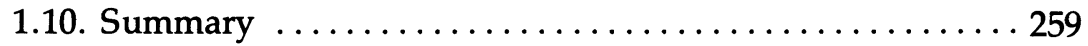

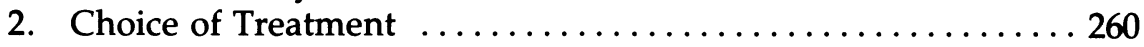

2.1. Conceptual Relevance of Treatment ............. 261

2.2. Effectiveness of Treatment $\ldots \ldots \ldots \ldots \ldots \ldots \ldots \ldots . \ldots \ldots 2$

2.3. Utilization of Treatment $\ldots \ldots \ldots \ldots \ldots \ldots \ldots \ldots \ldots 268$

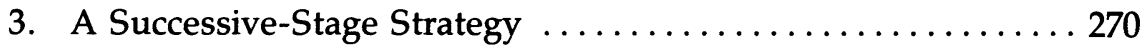

3.1. Identify and Conceptualize the Problem ......... 271

3.2. Formulate the Dependent Variables ............ 271

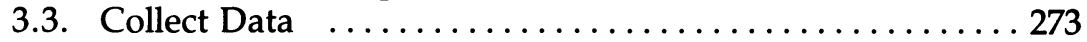

3.4. Formulate the Independent Variables $\ldots \ldots \ldots \ldots \ldots 274$

3.5. Disseminate Treatment ...................... 275

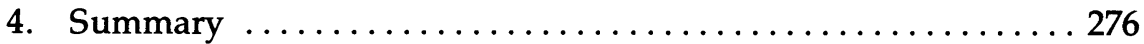

4.1. A Dissemination Technology: Two Choices? . . . . . . 277

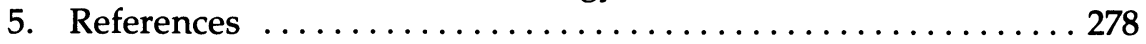

Drug Abuse by Children and Adolescents:

Perspectives on Incidence, Etiology,

Assessment, and Prevention Programming John J. Horan and Raymond P. Harrison

1. The Shifting Priorities of Drug Education $\ldots \ldots \ldots \ldots \ldots \ldots 285$

2. Prevalence of the Drug Problem $\ldots \ldots \ldots \ldots \ldots \ldots \ldots \ldots . \ldots \ldots$

2.1. Nicotine .............................. 287

2.2. Alcohol $\ldots \ldots \ldots \ldots \ldots \ldots \ldots \ldots \ldots \ldots \ldots \ldots \ldots \ldots . \ldots \ldots$

2.3. Marijuana $\ldots \ldots \ldots \ldots \ldots \ldots \ldots \ldots \ldots \ldots \ldots \ldots . \ldots . \ldots 289$ 
2.4. Hallucinogens $\ldots \ldots \ldots \ldots \ldots \ldots \ldots \ldots \ldots \ldots \ldots . \ldots \ldots$

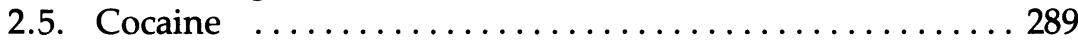

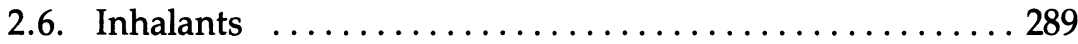

2.7. Amphetamines ........................... 290

2.8. Sedatives and Tranquilizers . ................. 290

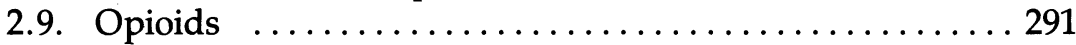

2.10. Concluding Observations on Usage Data ........... 291

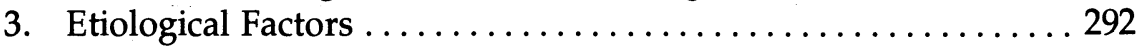

3.1. Behavior Variables ........................ 293

3.2. Classical Decision Theory and Drug Use ........... 295

4. Assessing the Outcomes of Drug Education: Conceptual and

Methodological Issues .......................... 296

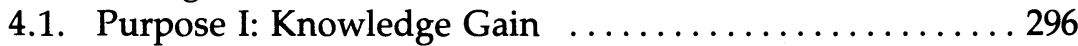

4.2. Purpose II: Attitude Change ................. 300

4.3. Purpose III: Behavior Change ................ 303

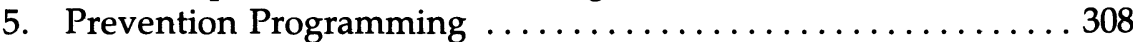

5.1. Information-Based Programming . .............. 309

5.2. Induced Cognitive Dissonance ................ 320

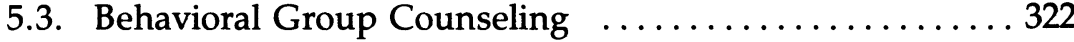

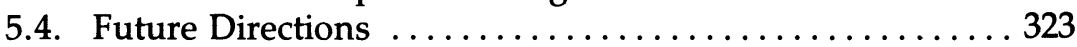

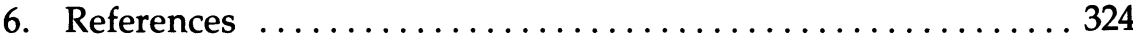

Genetics of Childhood Behavior Disorders David L. Pauls and Kenneth K. Kidd

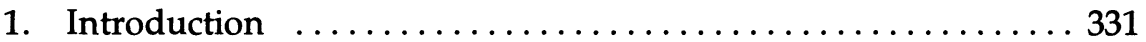

2. Etiologic Heterogeneity: A Possible Complication .......... 336

2.1. Selecting Homogeneous Groups by Inheritance Pattern . 337

2.2. Greater Homogeneity through Increased Diagnostic

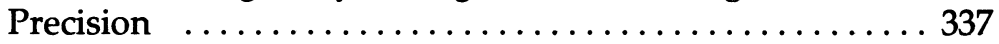

2.3. Greater Homogeneity through Sample Definition ..... 338

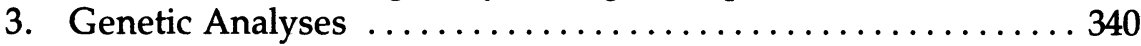

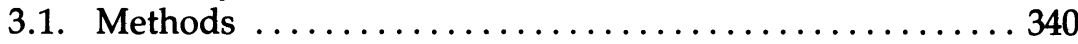

3.2. Examples of Genetic Analyses . .............. 344

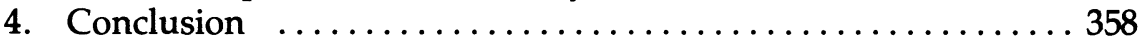

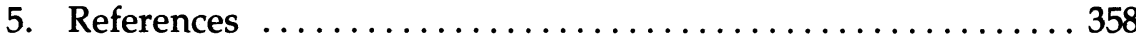

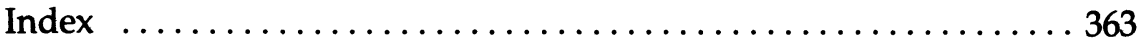

